\title{
EDITORIAL ESPECIAL
}

Recientemente, la Revista Latinoamericana de Investigación en Matemática Educativa - Relime, llevó a cabo un proceso de autoevaluación con la finalidad de establecer parámetros de desarrollo y localizar, de esta manera, zonas de oportunidad para el crecimiento internacional, con el fin de fortalecer su presencia mundial en la comunidad de Matemáticos Educativos. El estudio de los indicadores fue desarrollado por el equipo editorial de Relime, del cual se desprenden algunos datos de interés que hemos seleccionado, pues los consideramos relevantes para la confección de esta editorial.

Desde su fundación, Relime se concibió como un importante recurso científico comunitario, que se consolidaría a largo plazo, una estrategia de profundo aliento que le abriría un espacio a las diversas posturas teóricas para convivir y dialogar. Surgió entonces, entre nosotros, acostumbrados a vivir dentro de miradas teóricas totalitarias, la firme convicción de aprender a vivir en pluralidad ${ }^{2}$. Decíamos en esos años: mostrar al mundo que Latinoamérica existe y hacer pública la producción desarrollada para y desde Latinoamérica, lograr posicionar en el imaginario colectivo qué era lo que se investigaba en estas tierras y cuáles eran las problemáticas atendidas que reconocían las particularidades de nuestra región. Desde aquel tiempo, comenzando con el Volumen 0 y hasta el próximo Volumen 15, Número 2, se han publicado un total de 175 artículos de investigación sobre múltiples temáticas. La procedencia de los artículos es diversa y destacan principalmente de América y de Europa, así como algunas contribuciones de África y de Asia.

Del total de los artículos publicados, 36 son contribuciones españolas, 4 portuguesas, 3 francesas, 7 italianas, 1 danesa y 1 alemana. En el caso de América, tenemos 6 estadounidenses, 5 canadienses, 48 mexicanas, 14 argentinas, 4 chilenas, 4 colombianas, 6 cubanas, 1 peruana, 6 venezolanas. En cuanto África, tenemos una contribución sudafricana. Asimismo, existen 11 contribuciones realizadas por equipos autorales con miembros de distintos países, a las cuales hemos denominado mixtas, por ejemplo: Estados Unidos - Israel, México - Argentina, México - Colombia, México - España, Argentina - España, entre otras.

\footnotetext{
${ }^{2}$ GARNICA, A.V.M., FERNANDES, D.N. e SILVA, H. da. (2012). Entre a amnésia e a vontade de nada esquecer: notas sobre Regimes de Historicidade e História Oral. Boletim de Educação Matemática. 25(41), 213 - 250.
} 
Si bien hemos crecido significativamente en la cantidad y calidad de artículos publicados, debe considerarse que Relime tiene un factor de rechazo amplio, pues, en los últimos años, de cada 100 artículos recibidos sólo 43 son aceptados para su publicación.

Se ha cuidado escrupulosamente que los propios comités de Relime - Científico, de Redacción y Cartera de árbitros - tengan diversidad regional y representación temática y de género, a saber: los integrantes del Comité Científico son de Canadá, Argentina, Italia, Estados Unidos, Israel, Guatemala, México, Portugal, Cuba, Colombia, España, Francia y Reino Unido; en tanto, los miembros del Comité de Redacción pertenecen a los países de México, Brasil, Uruguay, Argentina, Chile, Costa Rica, China y Dinamarca. En cuanto a los miembros de la cartera de arbitraje, considerando a aquellos que han evaluado propuestas desde el 2009 a la actualidad, superan los 130 investigadores de diversas partes del mundo.

Con la base de datos del Journal Citation Report (JCR) del ISI, se muestran diversos indicadores bibliométricos relativos al papel de una revista en el concierto internacional de las publicaciones científicas. El factor de impacto (FI), como lo presentamos en (Cantoral, 2009) da un indicador del peso específico de una publicación entre sus pares, digamos que mide el promedio de citas por artículo en un periodo de dos años consecutivos. Dichas citas se obtienen sólo de revistas que estén, a su vez, incluidas en la misma base ISI.

En una editorial previa, explicamos ${ }^{3}$ :

Se define al parámetro A como el número de veces en que los artículos publicados en la Relime en el periodo 2007-2008 han sido citados por las publicaciones a las que se les da seguimiento a lo largo del 2009. Es decir, ISI considera fundamentalmente las citas en revistas que provengan de la misma base ISI.

Se cuenta el número de artículos publicados en el periodo 2007-2008, al cual nombramos como el parámetro B. El factor de impacto (FI) en el año 2009, en un periodo de dos años, es el cociente numérico de A entre B; es decir, FI = A / B.

En el JCR de 2011, Relime aparece por vez primera evaluada con un FI aproximado de 0.167, para el bienio 2009 - 2010. Este número, si bien es bajo, no lo es tanto si consideramos que se trata de su primer medición en esta base, es decir, está ubicada en el tercer tercio de las revistas en JCR, colocada, incluso, por encima de revistas de gran prestigio internacional, tanto inglesas como españolas, turcas, rusas y portuguesas. Sin duda, en los siguientes años, Relime irá en ascenso sólo en la medida en que las revistas del área de la Matemática

3 CANTORAL, R. (2009). Identidad y desarrollo: Matemática Educativa y Relime. Revista Latinoamericana de Investigación en Matemática Educativa 12(2), 145-150. 
Educativa incrementen sus citas a Relime, ¿cómo lograrlo desde Latinoamérica librando las barreras ideológicas y lingüísticas?

El Journal for Research of Mathematics Education - JRME, no aparece en este año en el JCR del ISI Web of Knowledge, aunque fue la primera revista de Matemática Educativa incluida en el JCR. Bolema-Boletim de Educação Matemática tiene un FI redondeado a 0, Enseñanza de las Ciencias - EC - tiene 0.188 y, más recientemente, Educational Studies in Mathematics - ESM - de 0.549, Revista Latinoamericana de Investigación en Matemática Educativa - Relime - de 0.167, Mathematical Thinking and Learning - MT\&L - de 0.393, International Journal of Science and Mathematics Education - IJSME - de 0.529.

Es sabido por la comunidad que Relime es una fuente indiscutible para la consulta, lectura y análisis de investigaciones innovadoras y de alto valor científico. Este posicionamiento lo ha ganado hace tiempo, y se observa, entre otras formas, a través del incremento de la cantidad de propuestas recibidas año tras año: 28 propuestas en 2007; 17 en 2008; 43 en 2009; 31 en 2010; 37 en 2011 y 57 en la primera mitad de 2012. Es decir, en los últimos años, más de 200 investigadores o grupos de investigación consideran a Relime como una de las principales revistas indicadas para hacer conocer a la comunidad, a nivel internacional, sus investigaciones; y otros miles más, acuden a ella para conocer las investigaciones de frontera. Es por ello que no nos resta más que preguntarnos, si tanto Relime como Bolema son revistas especializadas en Matemática Educativa, donde es posible publicar en castellano y portugués, y a su vez suelen ser consultadas por colegas latinoamericanos para llevar a cabo sus investigaciones, ¿cómo acostumbrarnos, editores, lectores y escritores de Relime a tener como umbral de normalidad el citar escritos de Bolema y viceversa? ¿Cómo podrá una publicación como el Educational Studies in Mathematics citar artículos que se publican en español o portugués tanto en Relime como en Bolema o en Enseñanza de las Ciencias? Nuevo dilema para la comunidad, dilema no trivial $^{4}$ (CANTORAL, 2012). Sabemos, por experiencias de colegas de otras disciplinas, la comunidad de físicos por ejemplo, que los científicos acuerdan acciones de grupo para mejorar su visibilidad internacional, como el pedir a los autores consultar sus artículos previos, o las publicaciones hechas en revistas cercanas, esto ayudaría a elevar la visibilidad de las revistas de nuestra

\footnotetext{
${ }^{4}$ CANTORAL, R. (2012). Nani gigantum humeris insidentes. Relime y el Acceso Abierto. Revista Latinoamericana de Investigación en Matemática Educativa 15(1), 5 - 7.
} 
región en ISI y en otros bancos de citas, de otro modo, el gran logro de tener algunas revistas incluidas en tan prestigioso índice se desvanecerá, sin más, en unos cuantos años.

Es por esto, que en esta editorial, queremos hacer un llamado a la comunidad: si bien está documentada la marcada tendencia en la cultura científica local de citar bibliografía anglosajona o francesa, al tiempo que no nos leemos entre nosotros, ni mucho menos nos citamos en nuestra propia lengua, ¿cómo lograr que las nuevas generaciones de investigadores en el campo se lean, se debatan, se citen en su propia lengua? Nuestra única respuesta es generando tradición, ojalá y tengamos tiempo.

Recapitulando... Luego del estudio que realizamos en la producción reciente de Relime podemos concluir varias cuestiones: que la comunidad de Relime se conforman por colegas de diversas partes del mundo, de manera explícita, mediante la colaboración en el comité editorial y el envío de contribuciones, y de manera implícita a través de su lectura; que es una de las principales fuentes de consulta para conocer la investigación de frontera; que la revista está posicionada dentro de los índices de mayor relevancia a nivel internacional; que el reconocimiento dentro de nuestra disciplina como una de las revistas de calidad para la publicación de investigaciones innovadoras es indiscutible; y que, por sobre todas las cosas, el crecimiento y la permanencia de nuestra revista, depende de nosotros mismos, la comunidad.

\section{Ricardo Cantoral \\ Daniela Reyes-Gasperini}

